\title{
Reasoning in Context-Aware Systems with Modal Logics
}

\author{
Yensen Limón ${ }^{1}$, Everardo Bárcenas ${ }^{1,2}$ and Edgard Benítez-Guerrero ${ }^{1}$ \\ 1 Universidad Veracruzana, Veracruz, México \\ zs16017367@estudiantes.uv.mx, edbenitez@uv.mx \\ 2 Consejo Nacional de Ciencia y Tecnología, Cd. de México, México \\ iebarcenaspa@conacyt.mx
}

\begin{abstract}
Context-aware systems are ubiquitous computing systems capable to adapt their behavior according to a dynamical changing environment. The development of reasoning and modeling techniques of context information have resulted a challenging task due to the inherent complexity of dynamical systems. In particular, modeling time and location context information have been so far constrained in current formalisms for context-aware computing due to expressive and computational limitations. Due to the well-known balance of expressive power and efficient reasoning algorithms associated to modal logics, we propose in the current work the use of expressive modal logics as a reasoning framework for context-aware pervasive systems. In particular, we describe a consistency model for a context-aware communication system. The consistency of this model is characterized in term of the satisfiability of the $\mu$-calculus (an expressive modal logic).
\end{abstract}

Keywords: Context-Aware Systems, Automated reasoning, Modal logics.

\section{Introduction}

Context-aware systems are flexible and adaptable to the context of information [6]. Due to the inherent huge amount of context variables in these systems, developing a context-aware system tends to be a very complex task for developers. In order to help reduce complexity and improve the maintenance and scalability in context-aware systems, it then is necessary to use modeling and reasoning techniques.

Basic modal logic $K$ is an extension of propositional logic with necessitation and possibility operators [7]. Many extensions of $K$, such as linear temporal logic (LTL), propositional dynamic logic (PDL), computational tree logic (CTL), description logics (DLs), etc., have been successfully used as reasoning frameworks in a wide rage of domains, such as program analysis, knowledge representation, databases, programming languages, etc. Due to the well-known balance of expressive power and efficient reasoning algorithms associated to modal logics [12], we propose in the current work the use of expressive modal logics as a reasoning framework for context-aware pervasive systems. 


\subsection{Motivations and Related Works}

One of the early approaches when modeling context aware systems is described in [10]. In this paper, it is described a context-aware communication system in terms of CML (object role modeling [9]) models. In particular, the input of the system consists of communication preferences for a set of persons and communication constraints for a set of locations. The system outputs a ranked set of communication channels suggestions satisfying preferences and location constraints. However, there is no guarantee the communication system is consistent, that is, whether communication preferences and location constraints allow any communication at all. In the current paper, we describe a consistency checker of the context-aware communication system. More precisely, we model the system in terms of $\mu$-calculus formulas, an expressive modal logic. Where if the formula is satisfiable, then the communication system is consistent, otherwise, the system is not consistent.

In [5], several spatial modeling approaches are described: set-based, hierarchical, graph-based, and hybrids. These approaches can model, with some limitations, several kind of spatial queries: position, nearest neighbor and range. One major limitation with these approaches is concerning time modeling. Since expressive modal logics have been successfully used as reasoning frameworks in the context of arithmetic constraints [3], which implies spatial modeling via euclidean distance, we believe that these expressive logics can also be used in the setting of spatial modeling, together with time modeling.

More recently, ontology-based modeling has gained a lot of attention in the pervasive computing community $[11,8,13]$. Ontologies are described in terms of the OWL(2) (Web Ontology Language) [2]. OWL(2) has been successfully studied and developed as a reasoning framework in the knowledge representation setting [1]. Since context information can be seen as a particular kind of knowledge, several proposed for context-aware modeling have been described, first in terms of the first version of OWL $[8,13]$, then with the last version OWL2 [11]. Although, OWL2 can model complex human activities, it still has some limitations with respect to spatial modeling. Since $\mathrm{OWL}(2)$ is in principle a Description Logic, which itself can be seen as member of the modal logic family [4], we believe extension of OWL2 with arithmetic constraints can be used for spatial modeling.

In Section 2, we describe the modal $\mu$-calculus, then in Section 3, we provide a consistency model for a context-aware communication system. Finally in Section 4, we give a brief summary of the paper and discuss further research perspectives.

\section{The $\mu$-calculus on Trees}

In this section, we introduce the $\mu$-calculus with converse, an extension of modal logic with and least and greatest fixed-points. Also converse modalities are included. Formulas are interpreted over finite unranked trees. 
Before definining the set of fomulas, we consider a fixed alphabet composed by three sets PROP, MOD and Var, where PROP is a set of proposition, $M O D=\{1,2,3,4\}$ is a set of modalities, and $\operatorname{Var}$ is a set of variables.

Definition 1 (Syntax). The set of $\mu$-calculus formulas is defined by the following grammar:

$$
\varphi::=p|X| \neg \varphi|\varphi \vee \psi|\langle m\rangle \varphi \mid \mu X . \varphi
$$

Where $p$ is a proposition, $m$ a modality, and $X$ is a variable.

We assume variables can only occur bounded and in the scope of a modality.

Formulas are interpreted as node subsets in unranked trees. We then now define tree structures in style of Kripke. A tree structure $K$ or simply a tree is defined as a tuple $(N, R, L)$ where:

$-N$ is a set of nodes;

- $R$ is a family of binary relations $R^{m}$ among nodes $(N \times N)$ forming a tree shape, that is, $R^{1}$ denotes the first child relation, $R^{2}$ the following (right) sibling, $R^{3}$ the parent, and $R^{4}$ the previous (left) sibling, we often write $n^{\prime} \in R(n, m)$ instead of $\left(n, n^{\prime}\right) \in R^{m}$; and

- $L$ is a function labeling $L: N \rightarrow 2^{P R O P}$.

We give a formal description of formula semantics.

Definition 2 (Semantics). Consider a tree $K$ and a valuation $V: \operatorname{Var} \rightarrow 2^{N}$. Formulas are interpreted as follows:

$$
\begin{aligned}
\llbracket p \rrbracket_{V}^{K} & =\{n \mid p \in L(n)\} \\
\llbracket \neg \varphi \rrbracket_{V}^{K} & =N \backslash \llbracket \varphi \rrbracket_{V}^{K} \\
\llbracket \varphi \vee \psi \rrbracket_{V}^{K} & =\llbracket \varphi \rrbracket_{V}^{K} \cup \llbracket \psi \rrbracket_{V}^{K} \\
\llbracket\langle m\rangle \varphi \rrbracket_{V}^{K} & =\left\{n \mid R(n, m) \cap \llbracket \varphi \rrbracket_{V}^{K} \neq \emptyset\right\} \\
\llbracket X \rrbracket_{V}^{K} & =V(X) \\
\llbracket \mu X . \varphi \rrbracket_{V}^{K} & =\bigcap\left\{N^{\prime} \mid \llbracket \varphi \rrbracket_{V[\mu X . \varphi / x]}^{K} \subseteq N^{\prime}\right\}
\end{aligned}
$$

A formula $\phi$ is satisfiable, if and only if, there is a tree such as the interpretation of $\phi$ over the tree is not empty, that is $\llbracket \phi \rrbracket_{V}^{K} \neq \emptyset$. If a tree $K$ satisfies a formula $\phi$, we say $K$ is a model of $\phi$.

Intuitively, propositions are used as labels for nodes, negation $(\neg)$ is interpreted as set complement, disjunctions are interpreted as set union. Modal formulas $\langle m\rangle \phi$ hold in nodes where there is an $m$-adjacent node supporting $\phi$. The least fixed-point is intuitively interpreted as a recursion operator.

Notation: $\phi \wedge \varphi:=\neg(\neg \phi \vee \varphi),[m] \phi:=\neg\langle m\rangle \neg \phi, \nu x . \phi:=\neg \mu x . \neg \phi[x / \neg x]$, $\top:=p \vee \neg p$, and $\perp:=\neg \top$.

Example 1. Consider for instance the following formula: $\langle 1\rangle p_{2} \wedge p_{1}$. This formula holds in nodes named $p_{1}$ with a child named $p_{2}$. In Figure 1 , there is a graphical representation of tree model for $\langle 1\rangle p_{2} \wedge p_{1}$.

The fixed-point operator is intuitively interpreted as a recursion operator, consider for instance $\mu X .\left(p_{3} \vee\langle 1\rangle X\right) \wedge\langle 1\rangle p_{2} \wedge p_{1}$. This formula holds in a $p_{1}$ node with a descendant $p_{3}$ and a child $p_{2}$. The tree in Figure 1 is also a model for $\mu X .\left(p_{3} \vee\langle 1\rangle X\right) \wedge\langle 1\rangle p_{2} \wedge p_{1}$. 


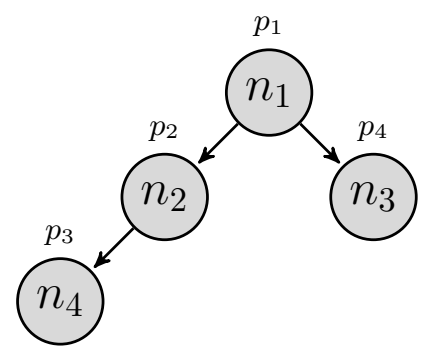

Fig. 1. Tree model for $\langle 1\rangle p_{2} \wedge p_{1}$ and $\mu X .\left(p_{3} \vee\langle 1\rangle X\right)$

\section{Consistency of a Context-Aware Communication System}

In this section, we describe a $\mu$-calculus characterization of a context-aware communication system [10]. The input of the communication system consists of a set of user communication preferences, a set of location communication constraints, and a schedule describing when users are at a specific location. Communication can be synchronous or asynchronous. For instance, let say the communication system is composed by two users, whose communications preferences consists of e-mail and chat, and e-mail and phone, respectively. e-mail and chat imply asynchronous communication, and phone implies synchronous communication.

It is then easy to see that user 1 can start communication asynchronously with user 2 . However, it may be the case that according to the schedule, user 2 will be located at places where e-mail is not available. Furthermore, user 2 may have access to e-mail but only before user 1 . In this two cases, we say the communication system is not consistent because constrains forbid communication. The $\mu$-calculus formula characterizing the system is satisfiable, if and only if, the system is consistent.

Formally, we describe the input of the system as sets.

\section{Definition 3 (System input).}

- The set of communication channels $C H=\left\{c_{1}, \ldots, c_{n}\right\}$ is divided in asynchronous and synchronous, that is, $C H=A C \cup S C$ and $A C \cap S C=\emptyset$.

- The set of user preferences (restrictions) PC is composed by pairs $(p, c)$, Where $p$ is a user proposition, and $c$ is a communication channel.

- The set of communication constraints for locations PL is composed by pairs $(l, c)$, where $l$ is a location proposition and $c$ is a communication channel.

- The schedule is defined as the following set: $S C H=\{(p, t, l) \mid p$ is a user proposition, $t$ is a time proposition, $l$ is a location proposition $\}$.

In order to semantically define the consistency of the communication system, we define a consistency model.

Definition 4 (System consistency model). Given a set of communication channels $C H$, a set of user preferences $P C$, a set of location constraints $P L$, and 
a schedule $S C H$, we define the consistency model $C M \operatorname{Model}(C H, P C, P L, S C H)$ as a tree structure $(N, R, L)$, as follows:

- there is one node for the root $r \in L(n)$;

- one node for each time proposition $t_{i} \in L\left(n_{t_{i}}\right)\left(i=1, \ldots, k_{1}\right)$, which are the children of the root, that is, $R(n, 1)=n_{t_{1}}, R\left(n_{t_{j}}, 2\right)=n_{t_{j+1}}\left(j=1, \ldots, k_{1}-\right.$ 1);

- each time node has one child per location, that is, $l_{j} \in L\left(n_{l_{i, j}}\right)$ and $R\left(n_{t_{i}}, 1\right)=$ $n_{l_{i, 1}}$ and $R\left(n_{l_{j, s}, 2}\right)=n_{j, s+1}$, where $i=1, \ldots, k_{1}, j=1, \ldots, k_{2}$ and $s=$ $1, \ldots, k_{2}-1$

- each location node has one child per channel available, that is, $c_{s} \in L\left(n_{i, j, w}\right)$, $R\left(n_{l_{i, j}}, 1\right)=n_{c_{i, j, 1}}$ and $R\left(n_{c_{i, j, w}}, 2\right)=n_{c_{i, j, w+1}}$, where $i=1, \ldots, k_{1}, j=$ $1, \ldots, k_{2}, s=1, \ldots, k_{3}$ and $w=1, \ldots, k_{3}-1 ;$ and

- each channel node has one child per user with that channel preference, that $i s, u_{w} \in L\left(n_{c_{i, j, s}}\right), R\left(n_{c_{i, j, s}}, 1\right)=n_{u_{i, j, 1}}$ and $R\left(n_{u_{i, j, z}}, 2\right)=n_{u_{i, j, z}}$, where $i=$ $1, \ldots, k_{1}, j=1, \ldots, k_{2}, s=1, \ldots, k_{3}, w=1, \ldots, k_{4}$ and $z=1, \ldots, k_{4}-1$.

Intuitively, a consistency model is tree of 5 levels: the first level is the root; the second is composed by the time lapses considered in the schedule; the third level contains the locations; the communication channels available at a particular location compose the fourth level; and the last level contains the users associated to each communication channel. In Figure 2 it is depicted a graphical representation of a consistency communication model.

In order to define the consistency of the communication system, we define the following relations in a tree $(N, R, L)$ : a node $n_{1}$ is a child of a node $n_{2}$, written $\operatorname{child}\left(n_{1}, n_{2}\right)$, if and only if, $R\left(n_{1}, 1\right)=n_{2}$ or there is a non empty sequence $R\left(n_{1}, 1\right)=n_{1}^{\prime}, R\left(n_{1}^{\prime}, 2\right)=n_{2}^{\prime}, \ldots, R\left(n_{i}^{\prime}, 2\right)=n_{2}$; a node $n_{1}$ is a descendant of a node $n_{2}$, written descendant $\left(n_{1}, n_{2}\right)$, if and only if, there is a non empty sequence of nodes $n_{1}^{\prime}, n_{2}^{\prime}, \ldots, n_{i}^{\prime}$, such that $\operatorname{child}\left(n_{1}, n_{1}^{\prime}\right), \operatorname{child}\left(n_{1}^{\prime}, n_{2}^{\prime}\right), \ldots, \operatorname{child}\left(n_{i}^{\prime}, n_{2}\right)$; and a node $n_{2}$ is a following sibling of a node $n_{1}$, written $f \operatorname{sibling}\left(n_{1}, n_{2}\right)$, if and only if, $n_{1}$ is the same than $n_{2}$ or there is a (possibly empty) sequence of nodes $R\left(n_{1}, 2\right)=n_{1}^{\prime}, \ldots, R\left(n_{i}^{\prime}, 2\right)=n_{2}$.

Definition 5 (System consistency). Given a consistency model CModel, we say user $u_{1}$ can communicate with user $u_{2}$, if and only if,

- (Synchronously) there is a channel node $n_{c}$, such that $\operatorname{child}\left(n_{c}, n_{u_{1}}\right)$ and $\operatorname{child}\left(n_{c}, n_{u_{2}}\right)$, and there is a time node $n_{t}$, such that descendant $\left(n_{t}, n_{c}\right)$, descendant $\left(n_{t}, n_{u_{1}}\right)$ and descendant $\left(n_{t}, n_{u_{2}}\right)$;

- (Asynchronously) there is a channel node $c_{c}$, such that child $\left(n_{c}, n_{u_{1}}\right)$ and $\operatorname{child}\left(n_{c}, n_{u_{2}}\right)$, and there are two time nodes $n_{t_{1}}$ and $n_{t_{2}}$, such that $f \operatorname{sibling}\left(n_{t_{1}}, n_{t_{2}}\right), \quad$ descendant $\left(n_{t_{1}}, n_{c}\right), \quad \operatorname{descendant}\left(n_{t_{2}}, n_{c}\right)$, descendant $\left(n_{t_{1}}, n_{u_{1}}\right)$ and descendant $\left(n_{t_{2}}, n_{u_{2}}\right)$.

Synchronous communication among two users occurs in the system consistency model when the user nodes are children of the same communication channel node and descendants of the same time node. Asynchronous communication relax the time restriction: the user node who starts communication must be a 


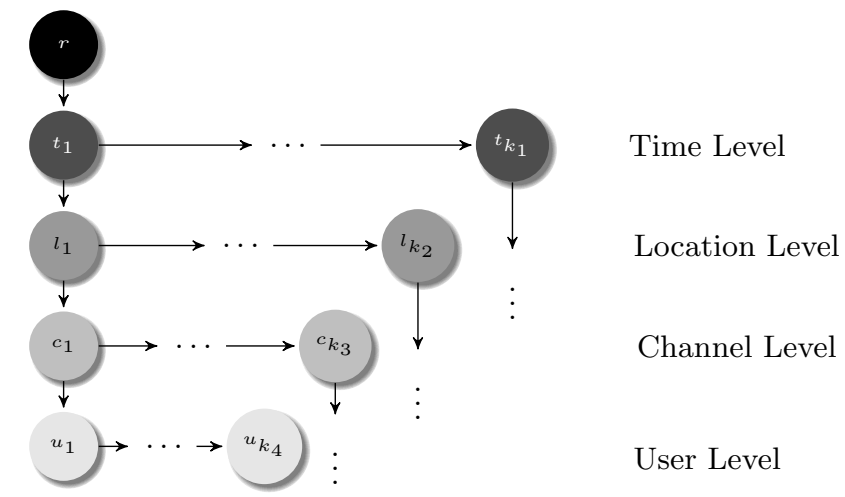

Fig. 2. Graphical representation of a consistency communication model

descendant of a time node, which is a previous sibling of the ancestor time node of the other user node.

We now define a logic formula that it is only satisfiable in a consistency model. To this end, we first define some notation: $\langle m\rangle^{0} \phi:=\phi,\langle m\rangle^{1} \phi:=\langle m\rangle \phi$, $\langle m\rangle^{i} \phi:=\langle m\rangle^{i-1}\langle m\rangle \phi$, where $m$ is modality and $i$ is the natural number.

We now describe a characterization of the consistency model in terms of a $\mu$-calculus formulas.

Definition 6 (Consistency formula). Given a system input $C H, P C, P L$ and $S C H$, and a communication type tc $\in\{$ syn,asyn $\}$, syn for synchronous and asyn for asynchronous, we define the consistency formula as follows:

$$
\begin{aligned}
& N_{1}(t c):=\langle 1\rangle\left(t_{1} \wedge N_{2}\left(t c, t_{1}\right) \wedge \bigwedge_{i=2}^{k_{1}}\langle 2\rangle^{i-1}\left(t_{i} \wedge N_{2}\left(t c, t_{i}\right)\right) \wedge \neg\langle 2\rangle^{k_{1}} \top\right) \\
& N_{2}(t c, t):=\langle 1\rangle\left(l_{1} \wedge N_{3}(t c, t, l) \wedge \bigwedge_{i=2}^{k_{2}}\langle 2\rangle^{i-1}\left(l_{i} \wedge N_{3}(t c, t, l)\right) \wedge \neg\langle 2\rangle^{k_{2}} \top\right) \\
& N_{3}(t c, t, l):=\left\{\begin{array}{cc}
\neg\langle 1\rangle \top & h(t c, t, l)=0) \\
F_{3}(t c, t, l) & h(t c, t, l) \geq 1)
\end{array}\right. \\
& F_{3}(t c, t, l):=\langle 1\rangle\left(c_{1} \wedge N_{4}\left(t c, t, l, c_{1}\right) \wedge \bigwedge_{i=2}^{h(t c, t, l)}\langle 2\rangle^{i-1}\left(c_{i} \wedge N_{4}\left(t c, t, l, c_{i}\right)\right)\right. \\
& \left.\wedge \neg\langle 2\rangle^{h(t c, t, l)} \top\right) \\
& N_{4}(t c, t, l, c):=\left\{\begin{array}{cc}
\neg\langle 1\rangle \top \quad k(t c, t, l, c, p)=0) \\
F_{4}(t c, t, l, c) & h(t c, t, l, c, p) \geq 1)
\end{array}\right. \\
& F_{4}(t c, t, l, c):=\langle 1\rangle\left(p_{1} \wedge \bigwedge_{i=2}^{k(t c, t, l, c, p)}\langle 2\rangle^{i-1} p_{i} \wedge \neg\langle 2\rangle^{k} \top\right)
\end{aligned}
$$


Where $k_{1}$ is the number of time propositions in the schedule, $k_{2}$ is the number of locations, $h(t c, t, l)=|\{c \in t c \mid(l, c) \in P L\}|$ is the number of communication channels of type $t c$ at a particular location $l$, and $k(t c, t, l, c, p)=\mid\{c \in t c \mid$ $(p, t, l) \in S C H,(p, c) \in P C\} \mid$ is the number of users with a communication channel $c$ as a preference.

Lemma 1. Given a system input channels, $P C, P L, S C H$, the consistency formula is satisfiable by the consistency model only, that is, $\llbracket N_{1}(t c) \rrbracket_{V}^{K} \neq \emptyset$, if and only if, $N$ is CModel.

We now define a characterization formula for each type of communication.

Definition 7 (Synchronous communication). The characterization formula $S C\left(u_{1}, u_{2}\right)$ corresponding to a synchronous communication from user $u_{1}$ to $u_{2}$ is defined as follows:

$$
\begin{aligned}
S C\left(u_{1}, u_{2}\right) & :=\bigvee_{i=1}^{k} F_{S C}\left(u_{1}, u_{2}, c_{i}\right) \\
F_{S C}\left(u_{1}, u_{2}, c_{i}\right) & :=\langle 1\rangle\left(\mu T .\left(\langle 1\rangle\left(F\left(u_{1}, c_{i}\right) \wedge F\left(u_{2}, c_{i}\right)\right) \vee\langle 2\rangle T\right)\right) \\
F(u, c) & :=\mu L .(\langle 1\rangle(\mu C .(c \wedge\langle 1\rangle(\mu P . u \vee\langle 2\rangle P)) \vee\langle 2\rangle C)) \vee\langle 2\rangle L)
\end{aligned}
$$

Where $k$ is the number of synchronous communication channels.

Definition 8 (Asynchronous communication). The characterization formula $A C\left(u_{1}, u_{2}\right)$ corresponding to a synchronous communication from user $u_{1}$ to $u_{2}$ is defined as follows:

$$
\begin{aligned}
A C\left(u_{1}, u_{2}\right) & :=\bigvee_{i=1}^{k} F_{A C}\left(u_{1}, u_{2}, c_{i}\right) \\
F_{A C}\left(u_{1}, u_{2}, c_{i}\right) & :=\langle 1\rangle\left(\mu T \cdot\left(\langle 1\rangle F\left(u_{1}, c_{i}\right) \wedge \mu T^{\prime} \cdot\left(\langle 1\rangle F\left(u_{2}, c_{i}\right) \vee\langle 2\rangle T^{\prime}\right) \vee\langle 2\rangle T\right)\right)
\end{aligned}
$$

Where $k$ is the number of asynchronous channels and $F$ is defined in Definition 7.

Now, from Lemma 1, it follows the consistency theorem.

Theorem 1. Given an input system channels, $P C, P L$ and $S C H$, user $u_{1}$ can communicate with user $u_{2}$ (according with Definition 5), if and only if,

- (synchronously) $N_{1}($ syn $) \wedge S C\left(u_{1}, u_{2}\right)$ is satisfiable;

- (asynchronously) $N_{1}$ (asyn) $\wedge A C\left(u_{1}, u_{2}\right)$ is satisfiable.

We now describe an example of the system input. We show in two cases when the system is consistent and inconsistent. 


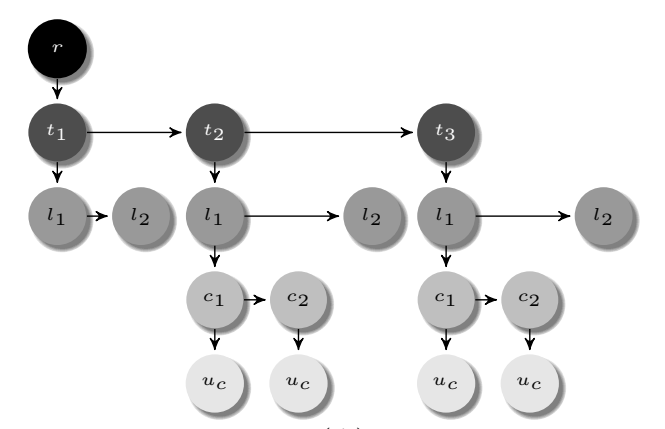

(A)

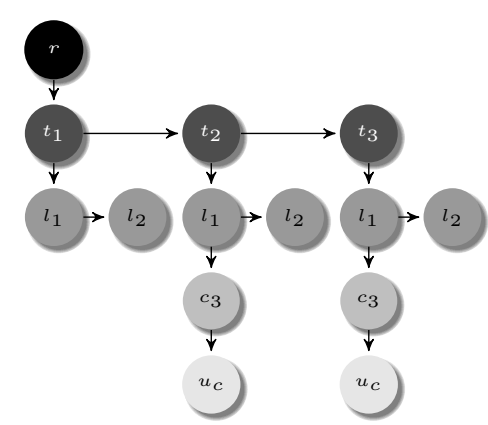

(B)

Fig. 3. The inconsistent CModel A is the synchronous communication, the formula is root $\wedge N_{1}(t c) \wedge S C\left(u_{c}, u_{y}\right)$ and the inconsistent CModel B is the asynchronous communication and the formula is root $\wedge N_{1}\left(t_{c}\right) \wedge A C\left(u_{c}, u_{y}\right)$. Where $r$ is the root, $c_{1}$ is the mobile call, $c_{2}$ is landline, $c_{3}$ is the message, $l_{1}$ is the office and $l_{2}$ is the warehouse

Table 1. Schedule of users within the company

\begin{tabular}{llll}
\hline \multicolumn{3}{c}{ Schedule } \\
\hline User Preferences & Location & Time \\
\hline$u_{y}$ & mobile call and message & Warehouse & $t_{1}, t_{2}$ and $t_{3}$ \\
$u_{c}$ & mobile call, landline and message & Office & $t_{2}$ and $t_{3}$ \\
\hline
\end{tabular}

Example 2. We consider the Table 1 where user $u_{y}$ arrives at $t_{1}$ to the warehouse and user $u_{c}$ arrives at $t_{2}$ to the office also this table shows user preferences and the location where they will be. Consistent system input.

- The set of communication channels is $C H=\{$ mobile call,landline, message $\}$.

- The set of user preferences is $P C=\left\{\left(u_{y}\right.\right.$, mobile call $),\left(u_{y}\right.$, message $),\left(u_{c}\right.$, mobile call $),\left(u_{c}\right.$, message $),\left(u_{c}\right.$, landline $\left.)\right\}$

- The set of communication constraints for location is $P L=\{($ Warehouse, mobile call), (Warehouse, message), (Office, mobile call), (Office, landline), (Office, message)\}

- The shedule is the following set: $S C H=\left\{\left(u_{y}, t_{1}\right.\right.$, Warehouse $),\left(u_{y}, t_{2}\right.$, Warehouse $),\left(u_{y}, t_{3}\right.$, Warehouse $),\left(u_{c}, t_{2}\right.$, Office $),\left(u_{c}, t_{3}\right.$, Office $\left.)\right\}$

Inconsistent system input. Now consider where the user $u_{y}$ has no preferences, we are based on the previous sets, the inconsistent system sets are as follows.

- The set of user preferences is $P C=\left\{\left(u_{c}\right.\right.$, mobile call $),\left(u_{c}\right.$, message $)$, $\left(u_{c}\right.$, landline $\left.)\right\}$

Example 3. We now present the $C M \operatorname{Model}(C H, P C, P L, S C H)$.

- The tree has a root (r).

- The time nodes are three $t_{1}, t_{2}$ and $t_{3}$ children of $r$.

- Each time node has two child nodes Office and Warehouse. 


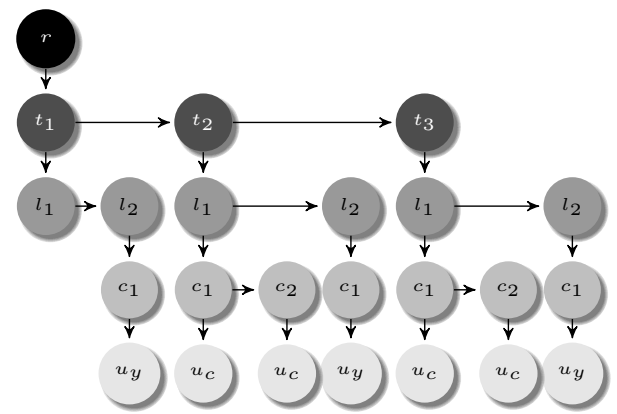

(A)

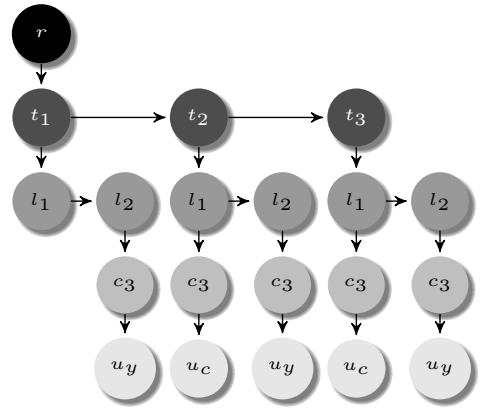

(B)

Fig. 4. The CModel A is the synchronous communication, the formula is $\operatorname{root} \wedge N_{1}(t c) \wedge$ $S C\left(u_{c}, u_{y}\right)$ and the CModel B is the asynchronous communication and the formula is root $\wedge N_{1}\left(t_{c}\right) \wedge A C\left(u_{c}, u_{y}\right)$. Where $r$ is the root, $c_{1}$ is the mobile call, $c_{2}$ is landline, $c_{3}$ is the message, $l_{1}$ is the office and $l_{2}$ is the warehouse

- Each location node has two synchronous channels (mobile call and landline) and an asynchronous channel (message).

- Each channel node has one user $\left(u_{y}\right.$ and $\left.u_{c}\right)$, which are essential to the user's preference.

In the Figure 4 it is observed the CModel for the consistent system with synchronous and asynchronous communication. Now in the Figure 3 is observed the CModel for the inconsistent system with synchronous and asynchronous communication. In this figure we observe that the user $u_{y}$ does not appear in the CModel, this is because he has not preferences.

Example 4. According with Definition 6, we present $N 1$ (syn) only one example when the system is consistent and uses a synchronous communication.

$$
\begin{aligned}
N_{1}\left(t_{c}\right):= & t_{1} \wedge N_{2}\left(t_{c}, t_{1}\right) \wedge\langle 2\rangle\left(t_{2} \wedge N_{2}\left(t_{c}, t_{2}\right)\right) \wedge\langle 2\rangle\langle 2\rangle\left(t_{3} \wedge N_{2}\left(t_{c}, t_{3}\right)\right) \\
& \wedge \neg\langle 2\rangle\langle 2\rangle\langle 2\rangle \top \\
N_{2}\left(t_{c}, t_{2}\right):= & \langle 1\rangle\left(l_{1} \wedge N_{3}\left(t_{c}, t_{2}, l_{1}\right) \wedge\langle 2\rangle\left(l_{2} \wedge N_{3}\left(t_{c}, t_{2}, l_{2}\right)\right) \wedge \neg\langle 2\rangle\langle 2\rangle \top\right) \\
N_{3}\left(t_{c}, t_{2}, l_{1}\right):= & \langle 1\rangle\left(c_{1} \wedge N_{4}\left(t_{c}, t_{2}, l_{1}, c_{1}\right) \wedge\langle 2\rangle\left(c_{2} \wedge N_{4}\left(t_{c}, t_{2}, l_{1}, c_{2}\right)\right) \wedge \neg\langle 2\rangle\langle 2\rangle \top\right) \\
N_{4}\left(t_{c}, t_{2}, l_{1}, c_{1}\right):= & \langle 1\rangle\left(u_{c} \wedge \neg\langle 2\rangle \top\right)
\end{aligned}
$$

Where $c_{1}$ is the mobile call, $c_{2}$ is landline, $l_{1}$ is the office, $l_{2}$ is the warehouse and $t_{c}$ is syn.

Example 5. When a user $u_{c}$ wishes to contact the user $u_{y}$. Then, according to Definition 7 we present an example of synchronous communication.

$$
\begin{aligned}
F_{S C}\left(u_{c}, u_{y}, c_{1}\right) & :=\langle 1\rangle\left(\mu T .\left(\langle 1\rangle\left(F\left(u_{c}, c_{1}\right) \wedge F\left(u_{y}, c_{1}\right) \vee\langle 2\rangle T\right)\right)\right. \\
S C\left(u_{c}, u_{y}\right) & :=F_{S C}\left(u_{c}, u_{y}, c_{1}\right)
\end{aligned}
$$


Where $c_{1}$ is mobile call. This example shows the mobile call channel. This channel is the only in common for the two users. In this case it is possible to communicate between the two users. In the case where the user does not have preferences the system is inconsistent.

\section{Conclusions}

In this paper, we proposed the use of expressive modal logics as a reasoning framework of context-aware systems. In particular, we described a consistency model for a context-aware communication system $[10,6]$. This model is characterized in terms of the satisfiability of the modal $\mu$-calculus.

As further research perspectives, we believe extensions of the $\mu$-calculus, such as inverse programs, nominals and graded modalities, can be used to model time and location context information, a crucial expressive-computational bottleneck when reasoning in context-aware sytems [6].

\section{References}

1. Baader, F., Calvanese, D., McGuinness, D.L., Nardi, D., Patel-Schneider, P.F. (eds.): The Description Logic Handbook: Theory, Implementation, and Applications. Cambridge University Press (2003)

2. Baader, F., Horrocks, I., Sattler, U.: Description logics. In: Staab, S., Studer, R. (eds.) Handbook on Ontologies, pp. 21-43. International Handbooks on Information Systems, Springer (2009)

3. Bárcenas, E., Lavalle, J.: Global numerical constraints on trees. Logical Methods in Computer Science 10(2) (2014)

4. Bárcenas, E., Molero, G., Sánchez, G., Benítez-Guerrero, E., Mezura-Godoy, C.: Reasoning on expressive description logics with arithmetic constraints. In: 2016 International Conference on Electronics, Communications and Computers (CONIELECOMP). pp. 180-185. IEEE (2016)

5. Becker, C., Dürr, F.: On location models for ubiquitous computing. Personal and Ubiquitous Computing 9(1), 20-31 (2005)

6. Bettini, C., Brdiczka, O., Henricksen, K., Indulska, J., Nicklas, D., Ranganathan, A., Riboni, D.: A survey of context modelling and reasoning techniques. Pervasive and Mobile Computing 6(2), 161-180 (2010)

7. Blackburn, P., van Benthem, J.F., Wolter, F.: Handbook of modal logic, vol. 3. Elsevier (2006)

8. Chen, H., Perich, F., Finin, T.W., Joshi, A.: SOUPA: standard ontology for ubiquitous and pervasive applications. In: 1st Annual International Conference on Mobile and Ubiquitous Systems (MobiQuitous). pp. 258-267. IEEE Computer Society (2004)

9. Halpin, T.: Information Modeling and Relational Databases. The Morgan Kaufmann Series in Data Management Systems, Academic Press (2001)

10. Henricksen, K., Indulska, J.: Developing context-aware pervasive computing applications: Models and approach. Pervasive and mobile computing 2(1), 37-64 (2006)

11. Riboni, D., Bettini, C.: OWL 2 modeling and reasoning with complex human activities. Pervasive and Mobile Computing 7(3), 379-395 (2011) 
12. Vardi, M.Y.: Why is modal logic so robustly decidable? In: Immerman, N., Kolaitis, P.G. (eds.) Descriptive Complexity and Finite Models. DIMACS Series in Discrete Mathematics and Theoretical Computer Science, vol. 31, pp. 149-184. DIMACS/AMS (1996)

13. Wang, X., Zhang, D., Gu, T., Pung, H.K.: Ontology based context modeling and reasoning using OWL. In: 2nd IEEE Conference on Pervasive Computing and Communications Workshops (PerCom),. pp. 18-22. IEEE Computer Society (2004) 